\title{
Modélisation du niveau trophique des sols forestiers : évaluation du pH en fonction des groupements phytosociologiques
}

\author{
M de Tillesse, F Devillez \\ Université catholique de Louvain, unité des eaux et forêts, place Croix-du-Sud, 2, bte 9, \\ B-1348 Louvain-la-Neuve, Belgique
}

(Reçu le 27 septembre 1994 ; accepté le 17 février 1995)

\begin{abstract}
Résumé - La régénération des peuplements forestiers pose toujours au sylviculteur un premier problème, celui du choix des essences à privilégier ou à installer. Les critères de choix se sont précisés ces dernières décennies. Mais il reste la lourde tâche d'évaluer l'aptitude stationnelle. En ce qui concerne le niveau trophique des sols, le pH est une variable explicative d'une certaine importance. C'est aussi le casse-tête des pédologues quand il s'agit de le cartographier. La recherche d'une covariante telle que la végétation permet de résoudre le problème. Deux sources de données ont été utilisées. La première utilise des résultats d'expériences effectuées par NoirfaliseE (1984) sur le territoire belge. La deuxième a consisté à refaire, pour la Fagne de Chimay, une campagne de mesures de $\mathrm{pH}$. Les 2 voies suivies présentent une gradation logique des $\mathrm{pH}$ avec les niveaux trophiques attendus pour les différents groupements phytosociologiques. Les mesures de terrain font état d'une faible variation des $\mathrm{pH}$ au sein de types trophiques. Les coefficients de variation calculés restent inférieurs ou égaux à $5 \%$, ce qui est remarquable. En fin de compte, la phytosociologie nous permet de troquer la technique d'échantillonnage systématique, à taux de sondage élevé, contre celle de l'échantillonnage stratifié à taux de sondage réduit.
\end{abstract}

$\mathrm{pH}$ / sol forestier / phytosociologie / modélisation / aptitude stationnelle

Summary - Modelling of trophic levels in forest soils using phytosociology. The initial problem regarding the regeneration of forest stands faced by forestry experts is the choice of species to privilege or to be brought in. Now, the criteria are well known, but the task of evaluating site capacity remains. Soil pH is one of the best components to characterize trophic levels, but it is very difficult to map. Vegetation may solve the problem. Two methods were used. The first employed results of experiments carried out by Noirfalise (1984) in Belgium (tables II, III and IV), and the second consisted of resurvey of the $\mathrm{pH}$ measurements at La Fagne in Chimay (tables V, VI and VII). Both methods showed a logical $\mathrm{pH}$ gradation related to the trophic levels expected for the phytosiological groups (fig 2). Field measurements showed a low $\mathrm{pH}$ variation between trophic types. Variation coefficients remained inferior or equal to $5 \%$, which was significant. We suggest that phytosociology may be used to replace high intensity systematic sampling by low intensity stratified sampling. 


\section{INTRODUCTION}

Tous les phytosociologues s'accordent pour dire que les groupements végétaux sont la résultante de l'action simultanée, synergique ou antagoniste, des multiples facteurs du milieu. En Europe, les alliances et associations forestières sont suffisamment bien définies pour ne plus revenir sur le sujet. En revanche, de nombreux types phytosociologiques se différencient à l'échelle locale en fonction de subtiles variations du relief, du microclimat, du sol et des modes de gestion anciens ou actuels des peuplements. Là, nos connaissances doivent encore être perfectionnées. Ce sont justement ces différences qui intéressent le forestier tant pour apprécier la productivité potentielle (Noirfalise, 1984) que pour déterminer l'aptitude de la station pour telle ou telle essence.

L'historique des relations entre phytosociologues et forestiers est intéressante à considérer à cet égard ; elle a admirablement été dressée par Jacamon (1988). Depuis peu, la collaboration est devenue franche et le colloque phytosociologique de Nancy, en 1985, consacré à la foresterie le montre très bien. Grâce à cette meilleure intégration mais aussi aux progrès de l'informatique, l'étude des relations entre la végétation, le milieu abiotique, la gestion et la productivité forestière est maintenant réalisable sur une grande échelle. On en est ainsi arrivé au développement de nouveaux modes d'approche regroupés sous le terme de «typologie des stations forestières". Initiés vers la fin des années 1970 (Becker, 1977 ; Picard, 1979 ; Becker et al, 1980), les résultats acquis dans cette voie de recherche sont très bien résumés dans les articles de Becker (1988) et de Dume (1988). En Belgique, citons le travail de Thill et al (1988).

Mais on peut aller encore plus loin que la mise au point de méthodes typologiques de description des stations en insistant, tout comme Peltier (1988), sur le fait que la méthode phytosociologique sigmatiste, appliquée notamment aux forêts, doit être de plus en plus un outil devant servir à résoudre des problèmes écologiques bien précis.

C'est dans cet ordre d'idées que nous proposons le présent travail. II s'inscrit dans le cadre de la mise au point d'un modèle de prédiction du niveau trophique des sols forestiers en fonction d'indicateurs biologiques. Notre contribution cerne l'évaluation d'une des composantes pour l'estimation du niveau trophique : le $\mathrm{pH}$. La démarche se justifie du fait que les pédologues à l'origine de la carte des sols de la Belgique étaient orientés vers des applications plus agricoles que sylvicoles ; un certain nombre de paramètres utiles à l'évaluation du niveau trophique des sols forestiers ont donc été négligés. Plus précisément, la présente recherche est liée à la publication par la Région wallonne du Guide de boisement des stations forestières de Wallonie (Weissen et al, 1994).

\section{PROBLÉMATIQUE DE LA MESURE $\mathrm{DU} \mathrm{pH}$}

Le Guide de boisement, cité ci-dessus, présente les bases rationnelles du choix que le gestionnaire est appelé à faire. II propose une gamme d'essences de production bien adaptées aux conditions stationnelles des parcelles à reboiser. Un des points forts de la méthodologie de ce guide provient du fait que, à une exception près, les éléments nécessaires pour diriger le cheminement dans les clés de détermination des niveaux de productivité potentielle des stations sont des propriétés explicitement cartographiées, ou déductibles de cartes existantes (carte des sols et carte topographique de la Belgique). Il s'agit du profil pédologique, de la nature du substrat et de celle de la charge caillouteuse. Seule une estimation du $\mathrm{pH}$ des sols, au sujet de laquelle le Guide de boisement évoque l'intérêt de la phytoso- 
ciologie, est à acquérir en complément des informations déjà cartographiées. Cependant, la technique préconisée par Weissen et al (1994) est celle de la mesure du pHeau, qui a les avantages d'être facile, rapide et peu coûteuse. Par ailleurs, suite à une étude en Fagne de Chimay, Goovaerts et al (1989) concluent à une plus grande fiabilité de la mesure de ce paramètre par rapport à celle d'autres paramètres qui contribuent à estimer le niveau trophique des sols tels que la conductivité, la capacité d'échange cationique, l'acidité d'échange, le taux de $\mathrm{Ca}^{++}$, etc.

Malheureusement, la mesure du pH présente plusieurs inconvénients.

D'une part, Goovaerts et al (1989) montrent que le $\mathrm{pH}$ des sols forestiers varie sensiblement d'un endroit à l'autre et d'une saison à l'autre sur un même site ; il en résulte une faible fiabilité des mesures, ainsi qu'une importante difficulté d'interprétation des résultats. La variation spatiale de cette mesure est très grande ; la distance au-delà de laquelle les observations deviennent spatialement indépendantes est de l'ordre de $4 \mathrm{~m}$. Plus récemment, dans le Bois-SaintGeorges à Chimay, Thierron et Laudelout (1994) ont réalisé des séries de mesures distribuées en structure gigogne, de mètre en mètre, de 10 en $10 \mathrm{~m}$, de 25 en $100 \mathrm{~m}$ et de 100 en $100 \mathrm{~m}$, totalisant plus de 1500 prélèvements. Leurs conclusions sont pratiquement identiques à celles des auteurs précédents. On voit que, pour être finement représentative des réalités de terrain, une carte des $\mathrm{pH}$ devra être faite sur la base d'un échantillonnage très serré. La mesure de $\mathrm{pH}$ devient un problème préoccupant lorsque l'on passe du niveau d'une parcelle au niveau d'une série entière dont il faut évaluer l'aptitude des stations.

Ces inconvénients nous conduisent à proposer une méthode alternative, qui réside dans l'appréciation du $\mathrm{pH}$ par des indicateurs biologiques. Encore faut-il pouvoir mener cette appréciation d'une manière simple et rapide. C'est cette possibilité qui est envisagée ici, par le biais de l'informatique et du traitement des données dans un langage relationnel.

\section{MATÉRIEL ET MÉTHODES}

Le site expérimental est celui de la Fagne de Chimay, en Belgique, et plus particulièrement des forêts domaniales du Bois-Saint-Georges et du Bois-Robert. L'altitude varie de 215 à $280 \mathrm{~m}$. À part les alluvions modernes des fonds de vallées et les quelques dépôts limoneux des plateaux et des pentes douces, la lithologie est dominée par les assises schisto-psammitiques du Famennien. L'altération de ce matériau parental est à l'origine d'une texture argileuse. Les sols caillouteux sont largement majoritaires ; ils résultent du mélange des produits d'altération de la roche-mère et, par endroits, des limons déposés au quaternaire. $\mathrm{Ce}$ sont des sols bruns (à horizon B structural). Ils sont lourds et se caractérisent par un régime hydrique alternatif : gorgés d'eau en hiver et secs en été.

L'étude utilise les relevés de végétation effectués par Devillez et Delhaise (1986). Elle se fonde sur la carte de végétation levée par ces mêmes auteurs en ce qui concerne le Bois-Saint-Georges et une nouvelle carte levée en 1993 pour le BoisRobert. Les individus des groupements recensés se rattachent au Pulmonario-Carpinetum décrit par Sougnez (1978) à propos de la chênaie-charmaie édaphique famennienne. Classées par ordre de fraîcheur croissante, 3 sousassociations peuvent être distinguées : typicum, deschampsietosum et filipenduletosum. Au niveau inférieur, une subdivision basée sur les exigences trophiques des espèces permet de différencier 3 variantes : oligotrophe, mésotrophe et eutrophe. Enfin, un faciès à Luzula sylvatica (Huds) Gaud peut être distingué dans la variante oligotrophe et un faciès à Primula elatior $(L) L$ peut être distingué dans le deschampsietosum (Delhaise et al, 1986).

Pour attribuer une valeur chiffrée de $\mathrm{pH}$ à chaque groupement rencontré, 2 voies possibles ont été explorées. La première consiste à utiliser la banque de données existante constituée par Noirfalise (1984) pour la Belgique. La deuxième voie consiste à attribuer les valeurs de $\mathrm{pH}$ issues d'une nouvelle campagne de récolte de données 
via un sondage sur la base d'une stratification phytosociologique du domaine d'étude.

\section{La banque de données existante}

Une banque informatisée de données a été constituée en encodant le Répertoire écologique de la flore vasculaire des forêts belges (Noirfalise, 1984). Sous forme de sigles, on dispose, pour chaque espèce citée, des informations concernant : le groupe socio-écologique, la forme biologique, l'appartenance phytogéographique, les exigences trophiques, les exigences hydriques et les exigences photiques. Les exigences trophiques ont été traduites, toujours selon les indications du même auteur, en valeurs chiffrées sous forme d'une fourchette des $\mathrm{pH}$.

On s'est heurté à la difficulté de caractériser par des chiffres les espèces couvrant plusieurs catégories trophiques comme, par exemple, les acidophiles à neutroclines. Dans certains cas ce genre d'appellation signifie que l'espèce couvre toute l'amplitude des catégories désignées ; dans d'autres cas il s'agit d'une espèce dont les exigences trophiques sont intermédiaires. Faute de pouvoir décider a priori, il a été décidé d'encoder les 2 possibilités. Sous la rubrique «amplitude large", on a reporté les valeurs extrêmes des catégories citées, c'est-à-dire le minimum de la catégorie la plus faible et le maximum de la catégorie la plus élevée. Sous la rubrique "amplitude étroite", on a introduit les valeurs moyennes de ces catégories respectives.

\section{La végétation étudiée}

Dans la plupart des études actuelles de végétation, les tableaux phytosociologiques sont traités sur ordinateur à l'aide de logiciels de la catégorie des tableurs. Leur transfert sur une banque de données en SQL ne présente guère de difficultés. II est donc possible d'introduire aisément dans le modèle la liste des espèces répertoriées ainsi qu'une expression quelconque des coefficients moyens d'abondance-dominance pour chaque groupement mis en évidence. C'est ce qui a été fait pour le tableau phytosociologique synthétique présenté par Devillez et Delhaise en 1986 (tableau I).

Ce fichier se rapportant à une situation précise doit être complété par un champ supplémentaire d'attributs rattachant chaque espèce soit à un groupe d'espèces différentielles, soit à l'une ou l'autre des classes de fidélité suivantes: espèces constantes, indifférentes, accidentelles ou accessoires.

\section{Le traitement des données}

Le principe du traitement des données consiste à mettre en relation la banque de données de base au fichier décrivant la végétation du site considéré. Pour les espèces communes aux 2 fichiers, des algorithmes calculent les valeurs moyennes, minimales et maximales des exigences trophiques par groupe d'espèces différentielles. Les espèces constantes sont utilisées pour caractériser les groupements ne possédant pas d'espèces différentielles et généralement considérés comme "typiques". Les espèces indifférentes, accidentelles ou accessoires ne sont pas prises en compte. Les algorithmes globalisent toutes les espèces relevant d'un seul groupe de différentielles du moment qu'elles sont présentes dans au moins 1 des syntaxons d'ordre inférieur ou supérieur. Ainsi, par exemple, toutes les espèces différenciant une variante acide sont recherchées dans toutes les sous-associations et dans tous les faciès.

La programmation de ce modèle a été réalisée en SQL au sein du logiciel ORACLE. Appliquée à une fourchette de données, elle permet d'obtenir pour chaque groupement et par type d'amplitude, 9 types de valeurs :

- le minimum absolu, le minimum moyen et le maximum des minima (a) ;

- le minimum des moyennes, la moyenne des moyennes et le maximum des moyennes (b) ;

- le maximum absolu, le maximum moyen et le minimum des maxima (c).

Il est loisible de choisir entre la simple présence ou le coefficient d'abondance-dominance. Le deuxième cas ne diffère du premier qu'au niveau des calculs effectués sur la moyenne (b) des fourchettes de valeurs. Pour ce qui est des minima (a) et des maxima (c), la pondération n'a aucun sens et les résultats sont évidemment identiques. Les calculs sont effectués simultanément pour les amplitudes larges et les amplitudes étroites.

\section{Les pH observés en Fagne de Chimay}

Les valeurs estimées sur la base de la banque de données de Noirfalise doivent être confrontées 
Tableau I. Espèces différentielles des divers types trophiques du Pulmonario-Carpinetum (Devillez et Delhaise, 1986).

Oligotrophe Mésotrophe Eutrophe

Faciès typique

Faciès typique

Polytrichum formosum Hedw Teucrium scorodonia $\mathrm{L}$ Veronica officinalis $\mathrm{L}$ Pteridium aquilinum (L) Kuhn Galium saxatile $\mathrm{L}$ Solidago virgaurea $\mathrm{L}$ Leucobryum glaucum (Hedw) Angstr Hieracium $\mathrm{sp}$ Senecio fuchsii CC Gmel
Corylus avellana $\mathrm{L}$

Quercus robur L

Acer pseudoplatanus $\mathrm{L}$

Anemone nemorosa $\mathrm{L}$

Viola reichenbachiana Jord

Polygonatum verticillatum (L) All

Potentilla sterilis (L) Garcke

Poa nemoralis $\mathrm{L}$

Luzula pilosa (L) Willd

Fragaria vesca $\mathrm{L}$

Viola riviniana Reichb

Euphorbia amygdaloides L

Carex flacca Schreb

Poa chaixii Vill

Maianthemum bifolium (L) FW Schm

Scrophularia nodosa $\mathrm{L}$

Succisa pratensis Moench
Lamium galeobdolon $(\mathrm{L}) \mathrm{L}$

Glechoma hederacea $L$

Ranunculus ficaria $\mathrm{L}$

Paris quadrifolia $\mathrm{L}$

Stachys sylvatica $\mathrm{L}$

Arum maculatum $\mathrm{L}$

Dryopteris filix-mas (L) Schott

Acer campestre $\mathrm{L}$

Primula veris $\mathrm{L}$

Urtica dioica $\mathrm{L}$

Geranium robertianum L

Mercurialis perennis $\mathrm{L}$

Cirsium oleraceum (L) Scop

Brachypodium sylvaticum (Huds) Beauv

Stellaria holostea L

Alliaria petiolata (Bieb) Carava et Grande Allium ursinum $\mathrm{L}$
Faciès à luzule

Luzula sylvatica (Huds) Gaud

Deschampsia flexuosa $(\mathrm{L})$ Trin
Faciès à primevère

Primula elatior $(\mathrm{L}) \mathrm{L}$

Circaea lutetiana $\mathrm{L}$

Geum urbanum $\mathrm{L}$ à la réalité du terrain. Le principe adopté a été de mesurer le $\mathrm{pH}$ des sols au sein des groupements préalablement cartographiés en Fagne de Chimay. Il s'agit donc d'un protocole d'échantillonnage stratifié.

Des échantillons de sol ont été prélevés jusqu'à $10 \mathrm{~cm}$ de profondeur suivant la technique de l'échantillonnage composite : homogénéisation de 4 prélèvements à la sonde pédologique dans un rayon de $5 \mathrm{~m}$. La profondeur a été mesurée à partir de la partie supérieure de l'horizon organominéral. Dix échantillons composites ont été pris dans divers individus de chaque groupement, sauf pour le faciès à primevère, pour lequel l'échantillonnage s'est réduit à 5 prélèvements, vu sa faible représentativité dans le domaine d'étude.

En laboratoire, chaque échantillon composite a été dilué dans 2,5 fois son poids d'eau et le $\mathrm{pH}$ a été mesuré avec une précision théorique de
0,01 unité, qui est la limite de précision du $\mathrm{pH}$ mètre utilisé (WTW 90).

\section{RÉSULTATS ET DISCUSSIONS}

\section{La banque de données existante: les pH estimés}

L'application du programme réalisé au tableau phytosociologique de Devillez et Delhaise (1986) fait ressortir les variantes oligotrophes, mésotrophes et eutrophes ainsi que leurs faciès. Pour la variante mésotrophe, caractérisée justement par l'absence de différentielles, les espèces 
Tableau II. pH des divers types trophiques estimés (à partir de Noirfalise, 1984) en fonction de la présence des taxons indicateurs.

\begin{tabular}{|c|c|c|c|c|c|}
\hline & \multicolumn{2}{|c|}{ Oligotrophe } & \multicolumn{2}{|c|}{ Mésotrophe } & \multirow[t]{2}{*}{ Eutrophe } \\
\hline & À luzule & Typique & Typique & À primevère & \\
\hline \multicolumn{6}{|l|}{ Base du calcula } \\
\hline \multicolumn{6}{|l|}{ Minima } \\
\hline $\mathrm{pH}$ minimum & 3,5 & 3 & 3,5 & 4,5 & 4,5 \\
\hline $\mathrm{pH}$ moyen & 4 & 4 & 4,5 & 5,8 & 5 \\
\hline $\mathrm{pH}$ maximum & 4,5 & 4,5 & 5 & 7 & 5,5 \\
\hline \multicolumn{6}{|l|}{ Moyennes } \\
\hline $\mathrm{pH}$ minimum & 4 & 3,8 & 4,7 & 4,8 & 5,3 \\
\hline $\mathrm{pH}$ moyen & 4,5 & 4,5 & 5,6 & 6 & 6,3 \\
\hline $\mathrm{pH}$ maximum & 5 & 5,3 & 6,5 & 7 & 7,1 \\
\hline \multicolumn{6}{|l|}{ Maxima } \\
\hline $\mathrm{pH}$ minimum & 4,5 & 4,5 & 5,5 & 5,5 & 5,5 \\
\hline $\mathrm{pH}$ moyen & 5 & 5 & 6,5 & 6,3 & 6,8 \\
\hline $\mathrm{pH}$ maximum & 5,5 & 5,5 & 8 & 7 & 8 \\
\hline
\end{tabular}

Valeurs minimales, moyennes et maximales estimées sur la base des amplitudes larges. a Utilisation des valeurs minimales, moyennes ou maximales des fourchettes de $\mathrm{pH}$ fournies par Noirfalise (1984).

constantes ont été prises en compte. Les résultats sont résumés dans les tableaux II et III. Ils comparent les valeurs fournies par les amplitudes étroites et larges. De toute évidence, pour les amplitudes larges, les recouvrements entre les divers groupements sont considérables et les exigences écologiques sont dans tous les cas confondues. Les amplitudes étroites sont plus discriminantes et méritent donc d'être retenues. II ressort également que seule la prise en compte de l'exigence moyenne manifestée par chaque espèce répond aux objectifs fixés ici, à savoir cerner le mieux possible la gamme des $\mathrm{pH}$ compatibles avec les exigences des espèces différentielles choisies. On pouvait d'ailleurs s'attendre à de tels résultats eu égard aux principes régissant la constitution des groupes socio-écologiques. En effet, ils donnent une tendance moyenne résultant de la combinaison d'espèces dont les exigences écologiques se recouvrent dans une certaine zone, les minima ou les maxima de chaque espèce pouvant varier considérablement.

Le tableau IV compare les résultats obtenus en tenant compte de la simple présence d'une part, et des coefficients d'abondancedominance d'autre part. On y remarque que les différences sont faibles.

Dans la suite de ce travail, nous ne présenterons donc plus que les résultats des calculs effectués sur la présence-absence des espèces différentielles en utilisant uniquement les moyennes des exigences trophiques calculées sur base des amplitudes étroites. C'est ce mode de présentation (fig 1) qui traduit les moindres chevauchements entre les groupements testés. Tant les extrêmes des amplitudes écologiques (dési- 
Tableau III. pH des divers types trophiques estimés (à partir de Noirfalise, 1984) en fonction de la présence des taxons indicateurs.

\begin{tabular}{|c|c|c|c|c|c|}
\hline & \multicolumn{2}{|c|}{ Oligotrophe } & \multicolumn{2}{|c|}{ Mésotrophe } & \multirow[t]{2}{*}{ Eutrophe } \\
\hline & $\dot{A}$ luzule & Typique & Typique & À primevère & \\
\hline Base du calcul a & & & & & \\
\hline $\begin{array}{l}\text { Minima } \\
\text { pH minimum } \\
\text { pH moyen } \\
\text { pH maximum }\end{array}$ & $\begin{array}{l}3,5 \\
4 \\
4,5\end{array}$ & $\begin{array}{l}3 \\
4 \\
4,5\end{array}$ & $\begin{array}{l}3,5 \\
4,5 \\
5\end{array}$ & $\begin{array}{l}5 \\
5,6 \\
6,2\end{array}$ & $\begin{array}{l}4,5 \\
5 \\
5,5\end{array}$ \\
\hline $\begin{array}{l}\text { Moyennes } \\
\text { pH minimum } \\
\text { pH moyen } \\
\text { pH maximum }\end{array}$ & $\begin{array}{l}4 \\
4,5 \\
5\end{array}$ & $\begin{array}{l}3,9 \\
4 \\
4,5\end{array}$ & $\begin{array}{l}4,9 \\
5,5 \\
6,2\end{array}$ & $\begin{array}{l}5,2 \\
5,8 \\
6,5\end{array}$ & $\begin{array}{l}5,5 \\
6,3 \\
6,9\end{array}$ \\
\hline $\begin{array}{l}\text { Maxima } \\
\text { pH minimum } \\
\mathrm{pH} \text { moyen } \\
\mathrm{pH} \text { maximum }\end{array}$ & $\begin{array}{l}4,5 \\
5 \\
5,5\end{array}$ & $\begin{array}{l}4,5 \\
5 \\
5,5\end{array}$ & $\begin{array}{l}5,5 \\
6,5 \\
8\end{array}$ & $\begin{array}{l}5,5 \\
6,3 \\
7\end{array}$ & $\begin{array}{l}6,2 \\
6,9 \\
7,5\end{array}$ \\
\hline
\end{tabular}

Valeurs minimales, moyennes et maximales estimées sur la base des amplitudes larges. a Utilisation des valeurs minimales, moyennes ou maximales des fourchettes de $\mathrm{pH}$ fournies par Noirfalise (1984).

Tableau IV. $\mathrm{pH}$ moyens des divers types trophiques estimés (à partir de Noirfalise, 1984) sur la base des amplitudes étroites.

\section{Oligotrophe}

À luzule Typique

Base du calcul a

Présence

Abondance-dominance
4,5

4,6
Mésotrophe

Eutrophe

\begin{tabular}{lccccc} 
& Àluzule & Typique & Typique & À primevère & \\
& & & & & \\
\hline $\begin{array}{l}\text { Base du calcul a } \\
\quad \text { Présence }\end{array}$ & 4,5 & 4,5 & 5,5 & 5,8 & 6,3 \\
Abondance-dominance & 4,6 & 4,4 & 5,5 & 6 & 6,3 \\
\end{tabular}

Comparaison des résultats obtenus en tenant compte de la seule présence ou du coefficient d'abondancedominance des taxons indicateurs. a Utilisation des valeurs moyennes des fourchettes de $\mathrm{pH}$ fournies par Noirfalise (1984).

gnant respectivement les valeurs minimales et maximales rencontrées dans une liste donnée) que les valeurs moyennes s'échelonnent d'une manière logique, exception faite pour le groupement mésotrophe.
Enfin, sur base des indications fournies par les pH estimés, il n'y a aucune raison de séparer les faciès typique et à luzule du groupement oligotrophe. Ils ont donc été fusionnés. C'est un autre facteur qui devrait 


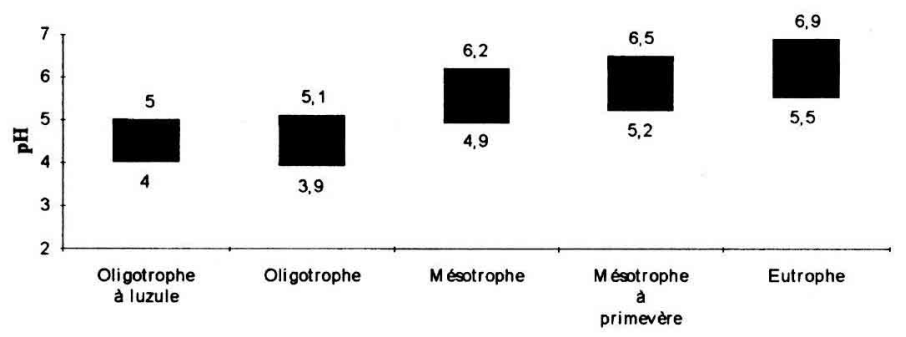

Fig 1. Distribution des valeurs estimées des $\mathrm{pH}$ en fonction des types trophiques du PulmonarioCarpinetum. expliquer l'extension sporadique de la grande luzule.

En tout état de cause, il est remarquable de constater, dès à présent, que les différents types trophiques du Pulmonario-Carpinetum s'échelonnent parallèlement à une échelle de $\mathrm{pH}$ moyens avec une précision moyenne de l'ordre d'une demi-unité.

\section{En Fagne de Chimay : les pH observés}

Le tableau $V$ présente l'ensemble des résultats obtenus, tandis que le tableau VI regroupe ces mêmes résultats par type trophique. On constate dans les 2 cas que les coefficients de variation ( $\mathrm{CV} \%$ ) calculés sont très faibles et donc que les $\mathrm{pH}$ observés varient peu à l'intérieur d'un groupement donné. Par ailleurs, on observe un gradient parfait des $\mathrm{pH}$ les plus acides pour les types oligotrophes aux plus neutres pour le type eutrophe. L'échelle établie est sensible puisque d'un palier à l'autre la variation des moyennes de $\mathrm{pH}$ ne va que de 0,1 à 0,8 unité.

Ces premières impressions sont entièrement confirmées par l'analyse statistique dont les résultats apparaissent au tableau VII. Comme pour les pH estimés sur base de la banque de données de Noirfalise (1984), il apparaît ici aussi qu'il n'existe aucune différence entre les types oligotrophes typique et à luzule. En revanche, les différences sont toujours significatives et le plus souvent hautement significatives entre toutes les autres catégories trophiques. Cela signifie clairement que les sous-associations et variantes du Pulmonario-Carpinetum sont un moyen efficace pour stratifier la Fagne de Chimay en zones

Tableau V. Effectifs, valeurs moyennes et coefficients de variation des pH observés dans le sol de tous les groupements du Pulmonario-Carpinetum.

\begin{tabular}{cccc}
\multicolumn{2}{c}{ Typicum } & Deschampsietosum & Filipendu- \\
Acide & Mésotrophe & Acide & Mésotrophe Eutrophe
\end{tabular}

$\begin{array}{lccccccccc}N & 10 & 10 & 10 & 10 & 10 & 10 & 5 & 10 & 10 \\ \text { Moyenne } & 4,58 & 4,59 & 4,70 & 4,62 & 4,64 & 4,74 & 5,52 & 6,30 & 6,15 \\ \text { CV }(\%) & 1,93 & 4,11 & 3,57 & 1,20 & 2,85 & 4,14 & 4,50 & 4,19 & 5,42\end{array}$


Tableau VI. Effectifs, valeurs moyennes et coefficients de variation des $\mathrm{pH}$ observés dans le sol des types trophiques du Pulmonario-Carpinetum.

\begin{tabular}{lccccc} 
& \multicolumn{2}{c}{ Oligotrophe } & \multicolumn{2}{c}{ Mésotrophe } & Eutrophe \\
& $\dot{A}$ luzule & Typique & Typique & $\dot{A}$ primevère & \\
& & & & & \\
$N$ & 20 & 20 & 30 & 5 & 20 \\
Moyenne & 4,60 & 4,61 & 4,72 & 5,52 & 6,22 \\
CV $(\%)$ & 1,63 & 3,41 & 3,56 & 4,50 & 4,86 \\
& & & & &
\end{tabular}

Tableau VII. Résultats de l'analyse de variance appliquée aux ensembles de $\mathrm{pH}$ observés pour discriminer les divers types trophiques du Pulmonario-Carpinetum.

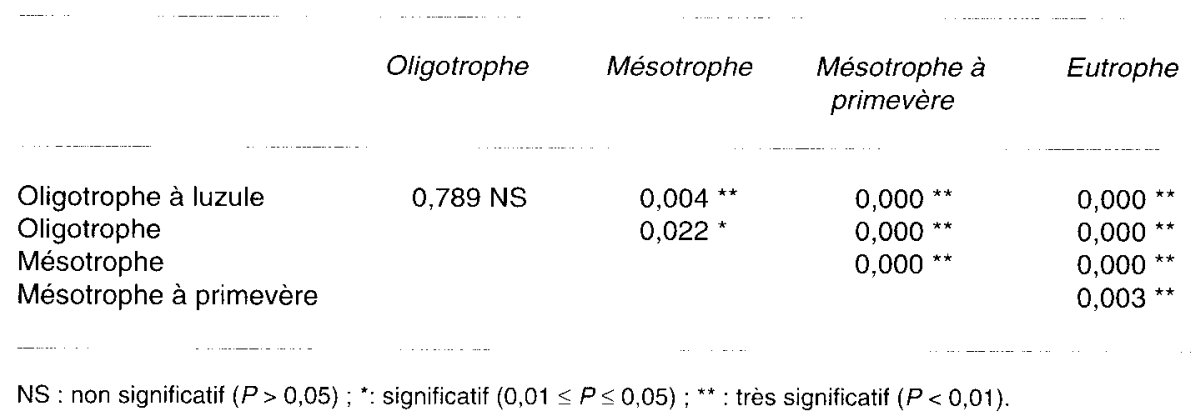

homogènes de $\mathrm{pH}$. Les valeurs observées au sein des différentes zones sont d'une cohérence surprenante.

La différence observée entre nos résultats et ceux de Goovaerts et al (1989), Thierron et Laudelout (1994), obtenus également en Fagne de Chimay, ne peut s'expliquer que par les méthodes d'échantillonnage mises en œuvre. En effet, ces auteurs ont appliqué un échantillonnage systématique, aveugle par définition, recoupant une mosaïque de groupements correspondant à des niveaux trophiques différents et tombant suivant les cas aux pieds des arbres, dans des espaces dégagés, des clairières, des traces de débardage... autant de facteurs pouvant expliquer une variation spatiale importante des résultats. Au contraire, notre méthode a cherché à caractériser chaque groupement en mesurant le $\mathrm{pH}$ dans des zones les plus homogènes possibles des peuplements, considérées comme typiques du point de vue phytosociologique. Ce point mérite d'être mis en évidence car il peut déboucher sur une méthode alternative d'inventaire des qualités des sols forestiers sur la base d'une carte de végétation préétablie.

\section{Comparaison entre les pH estimés et observés}

La figure 2 trace la relation entre les $\mathrm{pH}$ estimés sur la base des valeurs de la banque de données de Noirfalise (1984) et les $\mathrm{pH}$ observés au sein de chaque type trophique en Fagne de Chimay. 


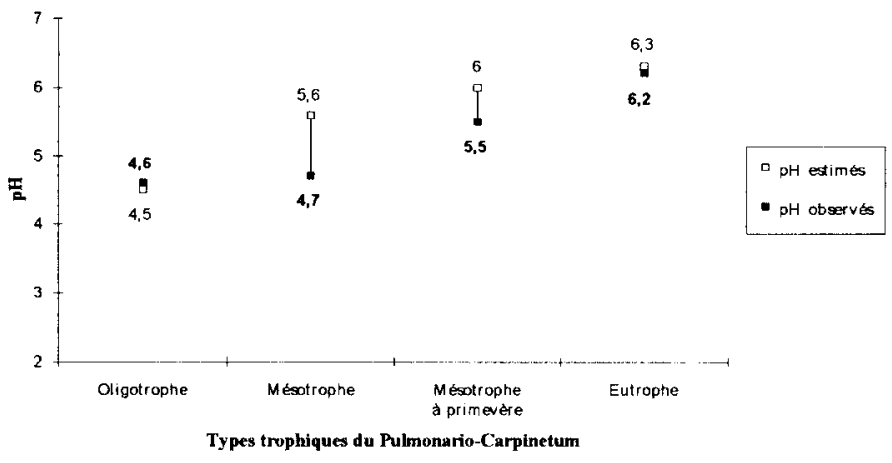

Fig 2. Mise en relation des valeurs de $\mathrm{pH}$ estimées et observées en fonction des types trophiques du PulmonarioCarpinetum.
Pour les types oligotrophe et eutrophe, les valeurs estimées et observées sont très proches les unes des autres. Pour les types de niveau trophique intermédiaire, les $\mathrm{pH}$ estimés sont supérieurs aux $\mathrm{pH}$ observés, mais avec un maximum d'écart de 0,9 unité entre les moyennes. L'écart moyen est, quant à lui, de 0,4 unité. Compte tenu de l'erreur commise lors des mesures et de la plus ou moins grande amplitude des exigences écologiques propres à chaque espèce ( 1 à 2 unités de $\mathrm{pH}$ ), ces écarts peuvent être considérés comme très faibles. Par ailleurs, l'existence de ces différences était parfaitement prévisible et normale. En effet, les groupements particuliers de la Fagne de Chimay n'avaient aucune raison de se situer exactement à l'optimum statistique d'une banque de données établie pour l'ensemble de la Belgique. Néanmoins, les $\mathrm{pH}$ observés se situent bien au sein des fourchettes de ces $\mathrm{pH}$ estimés pour chaque type trophique sur base des données de Noirfalise.

II reste que l'introduction des $\mathrm{pH}$ estimés dans la clé de détermination de l'échelle trophique de Weissen et al (1994) risque d'amener une erreur importante en ce qui concerne les niveaux trophiques moyens. En effet un pH estimé de 5,6 entraîne une valeur de 0 sur l'échelle trophique de Weissen et al (1994) ; alors que le $\mathrm{pH}$ observé n'est que de 4,72 , ce qui donne une valeur de 1 sur la même échelle trophique. Les conséquences pour l'aménagiste sont de taille car le rejet ou le choix d'essences importantes telles que le frêne commun, le chêne pédonculé ou l'érable sycomore en dépend. On peut conclure que la seule utilisation des $\mathrm{pH}$ estimés pour apprécier les niveaux trophiques est risquée, du moins dans le cas limite d'appréciation des classes trophiques 0 et 1 de Weissen et al (1994).

\section{CONCLUSIONS}

Comme le rappelle Becker (1973), la végétation est l'expression synthétique de l'ensemble des paramètres stationnels; elle en est la résultante. Si les caractéristiques physico-chimiques d'une station n'autorisent le développement que d'un groupement phytosociologique particulier, l'inverse montre que l'étude phytosociologique peut aboutir à évaluer directement certaines des caractéristiques physico-chimiques qui font les qualités trophiques d'un sol.

Dans cette recherche, nous nous sommes attachés à établir la relation entre les sous-associations et faciès du Pulmonario-Carpinetum et le $\mathrm{pH}$, en utilisant la classification établie par Sougnez (1978). Une étape importante dans l'élaboration d'un modèle de prédiction du niveau tro- 
phique des sols forestiers en fonction des groupements phytosociologiques est franchie. Pour compléter ce modèle, il resterait à établir la relation entre d'autres paramètres édaphiques jugés déterminants pour le développement des essences et la phytosociologie. L'origine de cette étude est liée à la publication par la Région wallonne du Guide de boisement des stations forestières de Wallonie (Weissen et al, 1994), qui préconise une mesure du $\mathrm{pH}$ en complément d'autres données pour l'évaluation du niveau trophique des sols forestiers, sans toutefois préciser de modalités d'échantillonnage. La technique d'échantillonnage systématique, utilisée classiquement, présente de sérieux inconvénients qui découlent de la faiblesse de la portée des mesures de $\mathrm{pH}$ en sols forestiers. La précision d'une carte des $\mathrm{pH}$ dépendra essentiellement du taux de sondage utilisé. Pour l'obtention de résultats fiables, ce taux de sondage doit être particulièrement élevé ; cela implique un temps de travail prohibitif quand il s'agit d'étendues forestières de plus de quelques dizaines d'hectares.

À l'opposé, l'évaluation du $\mathrm{pH}$ via l'étude phytosociologique semble d'une grande efficacité, du moins en ce qui concerne la Fagne de Chimay ainsi que le système de classification phytosociologique utilisé. Les mesures de terrain font état d'une faible variation des $\mathrm{pH}$ au sein des types trophiques. Les différences mises en évidence entre les ensembles de mesures comparant divers types trophiques sont significatives à hautement significatives. Les coefficients de variation calculés restent inférieurs ou égaux à $5 \%$. Cette homogénéité sousentend la qualité et la précision des résultats. En fin de compte, la phytosociologie nous permet de troquer la technique d'échantillonnage systématique à taux de sondage élevé, contre celle de l'échantillonnage stratifié à taux de sondage réduit. De plus, la perte de précision occasionnée lors du report cartographique des observations est minimale : les limites des groupements phytosociologiques observées sur le terrain sont les limites des zones de $\mathrm{pH}$. De plus, rappelons que l'étude phytosociologique d'une station est source de multiples autres renseignements, puisque la végétation spontanée se traduit comme une résultante écologique de l'ensemble des facteurs de l'environnement.

II reste encore des vérifications à faire sur d'autres associations, dans d'autres régions. L'analyse de la végétation se doit d'être complémentaire à celle de la pédologie. Signalons aussi que la méthode proposée n'est pas applicable aux parcelles plantées d'essences indigènes sciaphiles ou exotiques : maintenus denses, ces peuplements artificiels influencent la composition spécifique du sous-bois.

L'estimation des $\mathrm{pH}$ des divers groupements en fonction des caractéristiques écologiques de chaque espèce décrite dans le répertoire écologique de Noirfalise a également été tentée. Les meilleurs résultats sont obtenus par les calculs effectués sur les indices de présence-absence des espèces différentielles, en utilisant les moyennes des exigences trophiques calculées sur la base des amplitudes étroites. Néanmoins, si l'on injecte les valeurs estimées dans la clé de définition des aptitudes stationnelles de Weissen et al (1994), des erreurs importantes de choix d'essences peuvent découler de l'utilisation de ces $\mathrm{pH}$ estimés. Ces auteurs définissent également les exigences trophiques de chaque essence forestière. À ce propos, signalons que l'on pourrait discuter à l'infini des relations entre les exigences effectives des essences et l'échelle trophique établie. Seules de nouvelles observations ou expérimentations permettront de résoudre le problème. Des logiciels de géostatistique permettraient d'utiliser au mieux les banques de données phytosociologiques existantes combinées avec de nouvelles observations 
de terrain, pour lier potentiel nutritif du sol et exigences des essences. Le modèle élaboré au cours de cette étude constitue un premier pas dans cette voie.

\section{REMERCIEMENTS}

Nous tenons à remercier la Division de la nature et des forêts du ministère de la Région wallonne, qui a subsidié nos recherches. Nous adressons également nos plus vifs remerciements à $\mathrm{P}$ Hennebert, pour les remarques pertinentes apportées lors de la lecture du manuscrit et à JM Groux, pour son efficace collaboration technique.

\section{RÉFÉRENCES}

Becker M (1973) Écophytosociologie et production ligneuse. Ann Sci For 30, 287-306

Becker M (1977) Forêt française : pour une définition et une cartographie des stations. Bulletin technique de IONF 9, 9-19

Becker M (1988) Démarche méthodologique préconisée pour la typologie des stations forestières. $1 n$ : Colloq phytosociol: XIV Phytosociologie et foresterie (JM Jehu, ed), J Cramer, Berlin, Stuttgart, 299-311

Becker M, Le Tacon F, Timbal J (1980) Les plateaux calcaires de Lorraine. Types de stations et potentialités forestières. ENGREF, Nancy

Delhaise C, Devillez F, Laudelout H (1986) Étude du fonctionnement des écosystèmes forestiers et mise au point de modèles de gestion. Rapport, faculté des sciences agronomiques, université catholique de Louvain, Louvain-la-Neuve, Belgique

Devillez F, Delhaise C (1986) Groupements forestiers, sols et topographie dans la fagne de Chimay. in :
Colloq phytosociol: XIII Végétation et Géomorphologie (JM Jehu, ed), J Cramer, Berlin, Stuttgart, 553573

Dume $G$ (1988) Activités et résultats du groupe de travail sur la typologie des stations forestières. In: Colloq phytosociol : XIV Phytosociologie et foresterie (JM Jehu, ed), J Cramer, Berlin, Stuttgart, 289298

Goovaerts P, Gerard G, Frankart R (1989) Étude de la variabilité spatiale de quelques propriétés chimiques du sol en Fagne de Chimay, Belgique. Pédologie $39,191-207$

Jacamon M (1988) Historique des relations entre phytosociologues et forestiers en France. In : Colloq phytosociol: XIV Phytosociologie et foresterie (JM Jehu, ed), J Cramer, Berlin, Stuttgart, 1-9

Noirfalise A (1984) Forêts et stations forestières en Belgique. Les Presses agronomiques de Gembloux, faculté des sciences agronomiques de Gembloux, Gembloux

Peltier JP (1988) Remarques sur la méthode phytosociologique sigmatiste appliquée au milieu forestier. In : Collog phytosociol : XIV Phytosociologie et foresterie (JM Jehu, ed), J Cramer, Berlin, Stuttgart, 9397

Picard JF (1979) Une méthode de définition des stations en forêt : application à la forêt domaniale de Bellême. Ann Sci For 36, 211-229

Sougnez N (1978) Les chênaies-charmaies du district calcaire mosan. Centre d'écologie forestière et rurale, Gembloux 23, 1-85

Thierron V, Laudelout H (1994) Géostatistique de l'acidité du sol sous forêt feuillue. Colloque de l'Association française pour l'étude du sol, Lille

Thill A, Dethioux M, Delecour F (1988) Typologie et potentialités forestières des hêtraies naturelles de l'Ardenne centrale. IRSIA, Bruxelles

Weissen F, Bronchart L, Piret A, Lambert D (1994) Guide de boisement des stations forestières de Wallonie. Groupe interuniversitaire UCL, ULG, ULB et FSAGx 